\title{
Prototyping of a Low-Cost Stroboscope to Be Applied in Condition Maintenance: An Open Hardware and Software Approach
}

\author{
Laiany Brancalião ${ }^{1(\bowtie)}$, Caio Camargo ${ }^{1}$, José Gonçalves ${ }^{2}$, and José Lima ${ }^{2}$ \\ 1 Polytechnic Institute of Bragança, Bragança, Portugal \\ laianysuganuma@gmail.com, caioo.rafael@gmail.com \\ 2 Research Centre in Digitalization and Intelligent Robotics (CeDRI), Instituto \\ Politécnico de Bragança, Campus de Santa Apolónia, 5300-253 Bragança, Portugal \\ \{goncalves, jllima\}@ipb.pt \\ https://portal3.ipb.pt/index.php/pt/ipb
}

\begin{abstract}
This paper aims to develop a low-cost stroboscope, which consists of an optical equipment capable of generating flashes of light at different frequencies, allowing to measure the rotation velocity of machines and contributing to maintenance processes in the industry. This device is based on the stroboscopic effect, a visual event that occurs when a continuous movement is presented by a series of samples, generated by flashes of light. When the frequency of the rotation movement is the same frequency of light pulses, the process will appear stationary. Based on the high cost of the commercial stroboscopes, it was developed a stroboscope prototype based on the Arduino platform, LED technology and 3D printing with an open hardware and software. The final prototype went through calibration and validation processes, achieving a performance very similar to a commercial instrument.
\end{abstract}

Keywords: Stroboscope $\cdot$ Maintenance $\cdot$ Prototyping $\cdot$ Open source

\section{Introduction}

In the industry is very common the applying of resources and machines for implementation of the processes and creation of the products. For this reason, the measurement and control of such processes are essential to achieve better performance, efficiency, and consequently best quality, as well as, monitor the operation of the equipment, identify failures and ensure the security for the industry and the employees [1].

The measure instruments are the set of tools used with the objective of obtaining data about the particular processes. In general, these devices measure features like pressure, temperature, velocity, humidity, vibration, and are widely applied in machines such as motors, heaters, reactors, refrigerators, air conditioners, compressors, ovens, and other equipment. It is important to obtain the

(C) The Editor(s) (if applicable) and The Author(s), under exclusive license to Springer Nature Switzerland AG 2021

J. A. Gonçalves et al. (Eds.): CONTROLO 2020, LNEE 695, pp. 762-772, 2021.

https://doi.org/10.1007/978-3-030-58653-9_73 
periodic calibration of these instruments so that the results obtained through them be reliable and accurate [1].

The inspection and evaluation of equipment that work through continuous or periodic movements need to be done while it is in operation. Some devices such as automobiles, motors, propellers, textile looms, electric razors, blenders, and blowers [2], when they exhibit some defect, the best way to identify the problem is with the device turn on. However, this task becomes difficult when the movement is in high frequency.

The solution for this problem is the use of a stroboscope, an optical physical device that consists of the emission of blinking light in different frequencies desired, allowing the study of the velocity of an object and visualizing details of the movement that the human eyes fail to see [3]. Besides that, there is the possibility to do measurements without mechanical contact with the moving object, representing an advantage regarding traditional measurement methods [4].

Section 1.1 describes the stroboscopic effect, on which the device is based, the Sect.1.2 presents the analysis about the commercial stroboscopes available on the market, while the Sect. 1.3 is intended to the advantages of prototyping in $3 \mathrm{D}$ printing. The prototype description is presented in Sect. 2, including firmware and hardware. The stroboscope calibration is shown in Sect. 3, followed by the validation and results discuss in Sect. 4. Lastly, the conclusions and future work are presented in Sect. 5 .

\subsection{The Stroboscope and the Stroboscopic Effect}

This instrument is based on the stroboscopic effect, a visual phenomenon that happens when a blinking light source glows an object in movement, generating a sequence of samples. Depending on the blink light frequency, the process can seem forward or backward, so it is important to coincide the frequencies to see the stationary image, which enables the study and the maintenance of moving parts with high frequency [5].

A periodic movement repeats at equal time intervals, that is, it returns to the same position after complete a specific period. If a pulse of light illuminates a periodically moving object always when it is in a single position, the object appears stopped and the movement frozen [6].

Figure 1 represents an illustrative example of the stroboscopic effect through the use of a fan with a mark put on one of its propellers and a blink light source illuminating it. If the illumination frequency $\mathrm{f} 1$ is bigger than the frequency of movement $\mathrm{f} 2$, the body will appear to move backward, as presented in Fig. 1 a), if $\mathrm{f} 2$ is bigger than $\mathrm{fl}$, the body will appear to move forward, as in Fig. $1 \mathrm{~b}$ ), and if the frequencies coincide, the process will appear to be stationary, as in Fig. 1 c).

The stationary visualization of a rotate movement can also be observed if the flashes velocity are multiples or submultiples of the rotation velocity. If the flashing rate is twice the fundamental velocity of the machine, or also called second harmonic, two marks will be seen stopped in a distance of $180^{\circ}$. If the flashing 


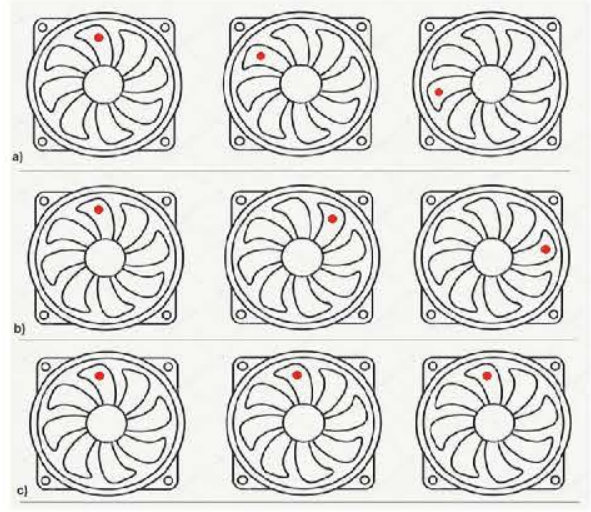

Fig. 1. Stroboscopic effect

rate is three times the fundamental velocity of the machine, third harmonic, three marks will be seen stopped in a distance of $120^{\circ}$ and so on [2].

When the light flashes velocity is half the rotation velocity, it will also seen one mark stopped, because the machine completes two rotations every flash. For this reason, to avoid errors measurements, it is recommended to start the velocity measurements at a high flash rate or the instrument's maximum flash rate, and then slow down until only one mark is seen [2].

For the visualization of the phenomenon, the light pulse length must be short enough compared with the periodic movement which intends to analyze, otherwise, image freezing may not be detected [6]. According to [6], the duty cycle must be below $10 \%$ of the total period [7], for the better visualization of frozen motion by the human eyes without blur.

Therefore, this exhibition of slow-motion provided by stroboscopic effect is useful to determine the angular velocity and analyze irregularities in machines with high velocity, because many times the problems can't be seen by the human eyes or the contact with the machine is very difficult and dangerous [2]. It's important to highlight the care that should be taken with this phenomenon, because depending on the situation, some moving parts can be seen as being stopped, of an undesirable way, causing accidents [7].

\subsection{Analysis of Existing Commercial Solutions}

Performing research about the stroboscopes commercially available nowadays, it could be found diverse industries that manufacture stroboscopes of many types. Basically, there are the stroboscope hand-held, the more compact called pocketstroboscope, the fixed and the high intensity ones, which have more than 1000 LEDs. The velocities ranges from 30 RPM to 500000 RPM, the light intensities varies from 3000 to $27000 \mathrm{~lx}$, and the prices range from $€ 200$ to $€ 3000$, depending on the model [8-10]. 
The modern electronic stroboscopes includes a light source and a voltage pulse generator [11], responsible for controlling the frequency of light flashes. The frequencies can be adjusted by the user through buttons until the stroboscope effect is observed, that is, until the object appears stopped. The current stroboscopes also include a display, where the information is shown, usually this information is the angular velocity in RPM.

Currently, most of the stroboscopes are digital and are manufactured using LED technology, but one still can be found the analog models. There are also found stroboscopes with xenon lamps, with built-in tachometers and those with high-intensity. In some device catalogs $[9,10]$ there is a recommended distance to the object of $20-50 \mathrm{~cm}$, the most stroboscopes found are powered by AA, Li-Ion or rechargeable batteries and all of them have a cold color temperature, around $6000 \mathrm{~K}[8]$.

\subsection{Prototyping in 3D Printing}

According to [13] 3D printing can be considered a technological revolution, it has gained the attention and interest of industries and research laboratories because this tool enables infinite possibilities [14] to design objects quickly, easily and creating physical prototypes with accuracy and precision [15].

3D printing is a technology applied to create three-dimensional objects through the successive deposition of layers of material and this is controlled digitally. This process is also called additive manufacturing, because objects are created adding, rather than removing material, like happened in subtractive manufacturing. Besides that, this kind of process reduces energy costs and material waste [14].

Nowadays, there are many kinds of materials that can be used for 3D printing, but the most common is plastic, being ABS, PLA, and Nylon. However, metal, ceramics, wood particles, and even chocolate have been used for it. The popularization and the constant cost savings of 3D printers has allowed the fabrication of several kinds of objects, such as, prototypes, tools, molds, prosthetics, toys, and among others [13].

\section{Prototype Description}

For the stroboscope prototyping, it was developed an electronics part, which included hardware and firmware, and a mechanical design. Figure 2 presents the systems diagram and the electronics components.

The LCD Keypad Shield performed as the interface, its buttons are the inputs for the Arduino Uno, which is responsible for processing all the information and generating two outputs. One of them is the velocity presentation on the display and the other is a digital modulated signal, which is sent to the driver circuit that switches the LEDs. The power supply for the stroboscope is a power bank, which one of its outputs power the Arduino board and the other power a boost converter that increases the voltage to power the driver circuit. 


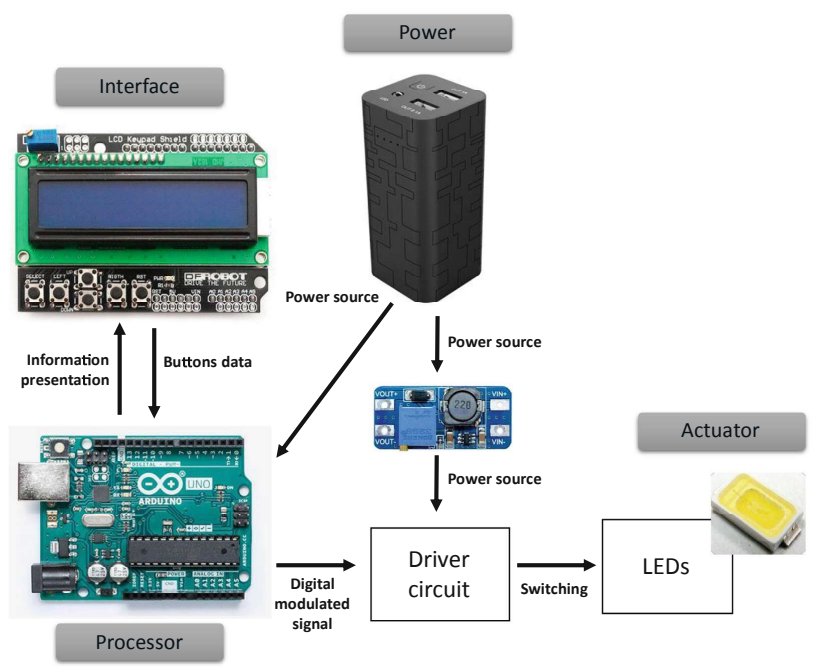

Fig. 2. Prototype components

The 3D model of the stroboscope design, presented in Fig. 3, was drawn using the SolidWorks, a 3D CAD software. The structure was elaborated to be as compact as possible and considering all the electronics components that would be assembled inside it.

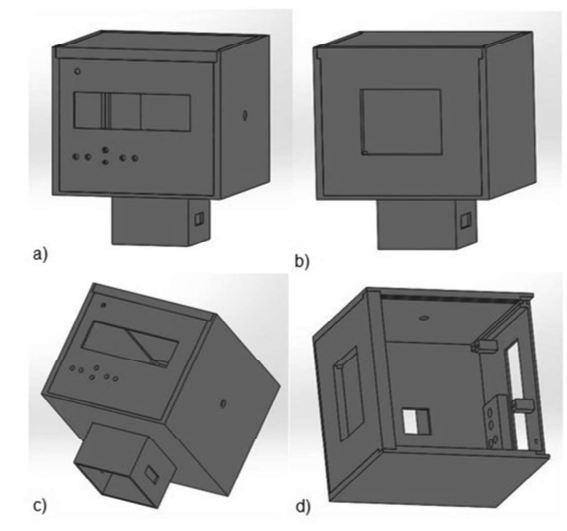

Fig. 3. Prototype 3D model a) front view b) back view c) bottom view d) top view

Figure 3 a) represents the front view of the stroboscope, intended to fix the LCD display and the buttons. Figure $3 \mathrm{~b}$ ) shows the back view, where the LEDs are exposed. Figure $3 \mathrm{c}$ ) corresponds to a bottom view, there is an extension where the power bank is dovetail with the stroboscope structure. A top view is 
presented in Fig. 3 d), without the lid, where it is possible to view the space for all the components.

\subsection{Firmware}

The firmware was developed using the Arduino Uno platform and based on three main tasks, buttons reading, angular velocity presentation on the display and generation of a digital modulated signal, as presented in Fig. 4. This signal must have a variable period and a fixed duty cycle, which represents the period that the signal keeps active. The duty cycle must be below $10 \%$ of the total period of the signal for the stroboscopic effect visualization without drag or blur $[6,7]$.

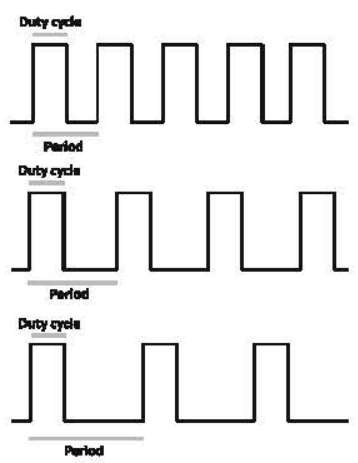

Fig. 4. Digital modulated signal

According to the frequency increase, the period tends to decrease and therefore, the value set for the duty cycle can become greater than $10 \%$ of the signal period. This way, when the duty cycle is close to overcoming $10 \%$ of the signal period, it is changed to a value below of that percentage. Therefore, the duty cycle is constant but not for all velocity values because it is necessary an adjustable duty cycle.

According to the frequency increase, the digital modulated signal generated by Arduino began to present a relative error significant. For this reason, it was established a velocity limit for the stroboscope prototype that ranges from 60 RPM to 100000 RPM with a duty cycle period in microseconds, which is adjusted according to high frequency values.

\subsection{Hardware}

For the hardware development, it was created a driver circuit, which included ten LEDs SMD5730 to act as the stroboscopic light, and a MOSFET responsible for switching the LEDs at different frequencies. This electronic component has 3 operation regions, cut, triode, and saturation, to perform as a switch it must 
work within the cut and triode regions [16]. This is possible sending the digital modulated signal from Arduino to the MOSFET gate.

The system was tested on the bench and then a PCB was created, which all the components were attached. The last step consisted to print the $3 \mathrm{D}$ model of the stroboscope prototype using a 3D printer. The parts went through the sanding and painting process in order to obtain the final result. Figure 5 a) presented the front view, where the LCD display and the buttons are attached. The back view can be seen in Fig. 5 b), where the LEDs are exposed behind a transparent plastic.

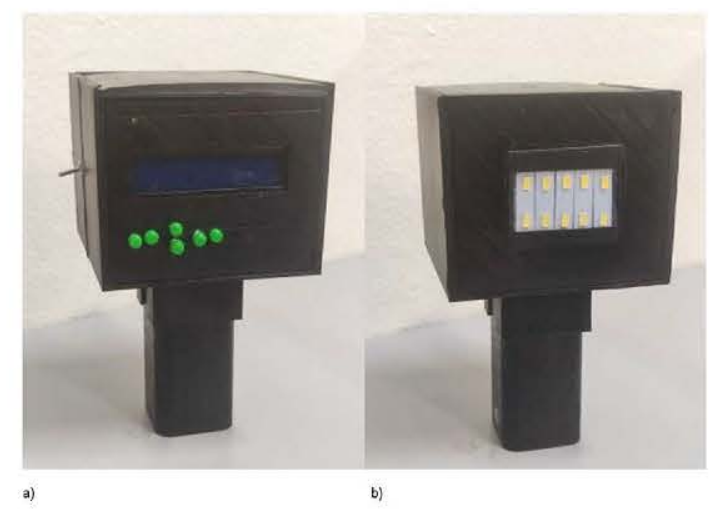

Fig. 5. Final prototype a) front view b) back view

The firmware developed in the Arduino IDE, driver circuit schematic and 3D model drawing created to develop the stroboscope prototype were available in a repository [17]. The final stroboscope prototype represents a lower cost compared to commercial stroboscopes presented in Sect.1.2, due to the use of low-cost electronic components and tools, such as Arduino and prototyping in 3D printing, which has become very popular due to cost and material savings, as presented in Sect. 1.3.

\section{Calibration Process}

The calibration of the stroboscopic light was based on an LDR sensor, a luminosity sensor that varies its resistance according to the intensity of light focused on it [18]. It was used a circuit composed of the sensor in series with a $10 \mathrm{k} \Omega$ resistor, powered by a $5 \mathrm{~V}$ DC source, and an oscilloscope connected over the resistor.

The stroboscopic light was positioned in front of the sensor and then, every light flash, the LDR resistance decrease, and the resistor voltage increase. Allowing to visualize the voltage pulses over the resistor, referring to the light flashes. 
Then, it was possible to measure the period between each flash and compare it with the digital modulated signal period.

In Fig. 6, the yellow signal represents the digital modulated signal sent by Arduino at 1000 RPM and the blue signal represents the light pulses captured by the sensor. The periods of both signals coincided with each other, confirming that the LED response was consistent with the signal sent by the microcontroller.

It is possible to observe that the voltage level of the blue signal didn't return to zero, because it wasn't possible to realize these measurements in a place totally dark, so the LDR always detected a little of luminosity. At high frequencies, above 10000 RPM, the calibration could not be done, because the LDR response isn't instantaneous, there is a latency time until the sensor answers by the transition from dark to light and vice-versa. Therefore, according to the frequency increase, the period decreases, and the transition is no longer clear, preventing the measurement of the period between each flash.

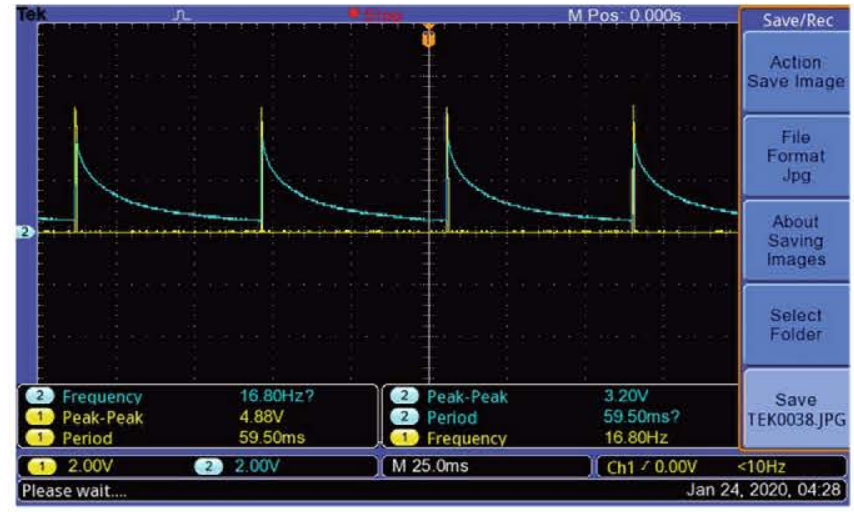

Fig. 6. Calibration - comparison between digital modulated signal and light pulses response.

\section{Validation of the Prototype and Results Discussion}

This chapter presents the validation tests to verify if the stroboscope prototype was able to measure the velocity of a rotating machine by visualizing the stroboscopic effect. The velocity of a three-phase motor, controlled in closed-loop and with a constant velocity, was measured using the stroboscope and a commercial tachometer. The measured results of both devices were compared in order to realize the validation of the stroboscope.

The results comparison between the stroboscope and the tachometer can be analyzed in Fig. 7, which shows the graphic of the devices' performance. The motor was subjected to different voltages and the graphic behavior shows the velocity increase according to the voltage increase and its stabilization around 
1500 RPM. Through this graphic, it is possible to notice that the results of both instruments remained aligned and close, with an error practically insignificant. These results are similar to those obtained in [12], in which the performance comparison between a low-cost tachometer and a commercial one is presented.

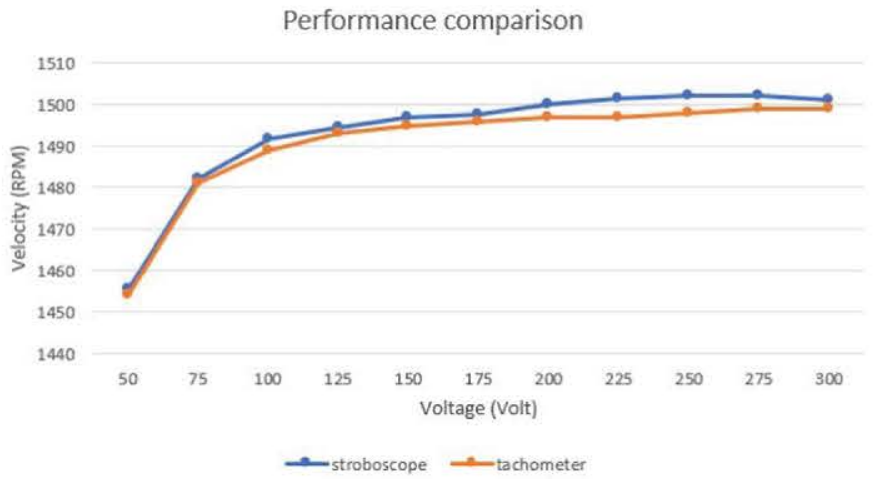

Fig. 7. Graphic of the performance comparison between stroboscope and tachometer.

The velocities measured by the stroboscope were obtained visually through the stroboscopic effect, that is, at the moment that the machine appears stopped. A white mark was done in the three-phase motor for the visualization of the phenomenon, which could be visualized successfully and without drag or blur. It is important to start the measurement process with the stroboscope in high frequencies or the maximum frequency of the device to have the assurance to find the right velocity.

Figure 8 a) was captured at the moment that the motor seemed to be stopped, that is, the moment that the rotation velocity of the motor coincides with the light flashes velocity of the stroboscope. In this situation each flash of light illuminates the mark always at the same position, when the motor completes one rotation, causing a frozen movement. Therefore, the velocity value of 1497.5 RPM, shown on the display, is the rotation velocity of the three-phase motor.

The stroboscopic effect can also be seen at velocities multiples of the real velocity. In Fig. 8 b) are seen two marks stopped because of the second harmonic, the flashes velocity is the double of the rotation velocity, that is, 2995 RPM. Then, at each rotation, the machine is illuminated by two flashes and as this process is very fast, in microseconds, the human eye does not notice this difference. The same situation occurs in Fig. 8 c), but in this case, it is the fourth harmonic, that is, the quadruple of the blinking velocity of light, 5990 RPM, so 4 marks are seen stopped. 


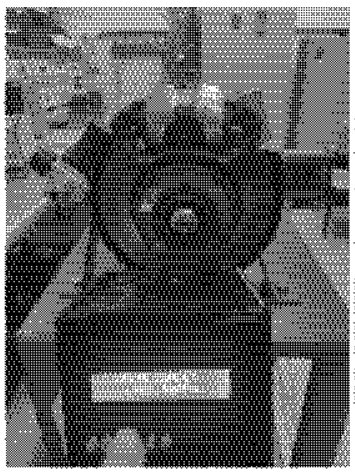

a)

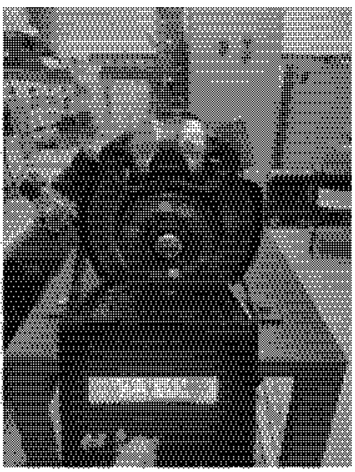

b)

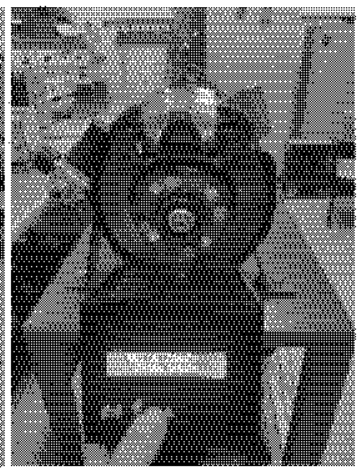

c)

Fig. 8. Stroboscopic effect visualization a) fundamental frequency b) second harmonic c) fourth harmonic

\section{Conclusions and Future Work}

This study contributed to the development of a stroboscope prototype, in which its performance is very similar to the commercial devices, and can be applied in condition maintenance. However, with some differences, such as low-cost, ergonomics, and open hardware and software, allowing any person that desires or needs this kind of equipment can develop their own stroboscopes.

The stroboscope prototype was able to generate light blinkings at different frequencies, allowing to measure the velocity of a rotate machine, through the stroboscopic effect visualization. Such an effect could be seen successfully and without drag, proving that the period used for the duty cycle of the modulated signal was good. Three-phase motor velocities measured by the prototype proved to be very close to the velocities obtained by the commercial tachometer.

The stroboscope behavior towards the user's action was shown plausible and the stroboscope worked as expected, providing the tools and techniques used to manufacture the prototype were enough. Through the calibration process with an LDR sensor, it was possible to compare the period of each light flash with the period of the digital modulated signal sent to the LEDs and both were consistent.

At high velocities, above $10000 \mathrm{RPM}$, the calibration process could not be done properly due to LDR sensor limitation, an alternative would be to use a faster light sensor, like a photodiode. As a way to improve the stroboscope, it is suggested as future work, the development of a communication interface, allowing to send the velocity value read on the display to a computer for registration or processing.

Acknowledgements. This work has been supported by FCT - Fundação para a Ciência e Tecnologia within the Project Scope: UIDB/05757/2020. 


\section{References}

1. A importância da Instrumentação. https://br.omega.com/prodinfo/instrumenta cao.html

2. Van Veen, F.: Handbook of Stroboscopy. GenRad Inc., Massachusetts (1977)

3. Mayer, V.V., Varaksina, E.I.: A stroboscopic light source for experiments in mechanics. In: Physics Education, vol. 52, pp. 1-7. IOP Publishing (2017). https:// doi.org/10.1088/1361-6552/aa8836

4. Vrcan, Ž., Lovrin, N., Gregov, G.: Primjene stroboskopa applications of stroboscopes. Eng. Rev. 29(1), 95-106 (2009)

5. Lai, H.W., Chow, M.W.K., Ma, C.K., Yan, A.Y.K.: Calibration of the frame rate of high-speed digital video recorders by stationary counting method: application of the stroboscopic effect. NCSLI Measure, 1-7 (2019). https://doi.org/10.1080/ 19315775.2019 .1572480

6. Kassamakov, I., Hanhijärvi, K., Aaltonen, J., Sainiemi, L., Grigoras, K., Franssila, S., Hæggström, E.: Stroboscopic white light interferometry for dynamic characterization of capacitive pressure sensors. J. Acoust. Soc. Am. 2523-2527. Proceedings European Conference on Noise Control (2002). https://doi.org/10.1121/1.2933663

7. Guedes, M.V.: Laboratório de Máquinas Elétricas - Estroboscopia. Faculdade de Engenharia da Universidade do Porto, Porto (2002)

8. Direct Industry - O salão online da indústria. https://www.directindustry.com/pt/ fabricante-industrial/estroboscopio-66061.html

9. Schmidt Control Instruments - Stroboscopes. https://www.hans-schmidt.com/en/ produkte/stroboscopes/

10. MonarchInstrument - Stroboscopes. https://monarchinstrument.com/collections/ stroboscopes

11. Mayer, V.V., Varaksina, E.I.: A LED stroboscope with computer control of light flashes. Phys. Educ. 53, 1-6 (2018)

12. Bakibillah, A.S.M., Uddin, M.A., Haque, S.A.: Design, implementation and performance analysis of a low-cost optical tachometer. In: IIUC Stud. 7, 107-116. (2011)

13. Rayna, T., Striukova, L.: From rapid prototyping to home fabrication: how 3D printing is changing business model innovation. In: Technological Forecasting and Social Change, vol. 102, pp. 214-224. Elsevier B.V. (2016). https://doi.org/10. 1016/j.techfore.2015.07.023

14. Ambrosi, A., Pumera, M.: 3D-printing technologies for electrochemical applications. Chem. Soc. Rev. 45, 2740-2755 (2016). https://doi.org/10.1039/c5cs00714c

15. Gross, B.C., Erkal, J.L., Lockwood, S.Y., Chen, C., Spence, D.M.: Evaluation of 3D printing and its potential impact on biotechnology and the chemical sciences. Anal. Chem. 86, 3240-3253 (2014). https://doi.org/10.1021/ac403397r

16. Sedra, A.S., Smith, K.C.: Microeletrônica. Oxford University Press, Pearson (2007)

17. Repository - stroboscope files. https://github.com/Laiany/stroboscopeLaiany

18. Sunrom Technologies: Light Dependent Resistor - LDR, July 2008 